\section{Non-alcoholic fatty liver disease: Update on treatment options and translational implications of sleep disruption}

\author{
Steve M D’Souza, Byung S Yoo, Kevin V Houston, Ankit J Patel, \\ Parth J Parekh and David A Johnson*
}

Department of Internal Medicine, Division of Gastroenterology, Eastern Virginia Medical School, Norfolk, VA, USA

\section{Abstract}

Non-alcoholic fatty liver disease (NAFLD) is a condition that is associated with cirrhosis and hepatocellular carcinoma, and is increasing in prevalence worldwide. Sleep disruptions are commonly seen in NAFLD, and the disease process is associated with sleep disorders, including obstructive sleep apnea, circadian rhythm disorders, and insufficient sleep. The intermittent hypoxia seen in obstructive sleep apnea may contribute to fibrotic changes in the liver.

A major component of this linkage may be related to gut microbiome changes. One notable change is increase in Bacteroidetes/Firmicutes ratio, and decrease in flora that ferment fiber into anti-inflammatory short-chain fatty acids. Several therapeutic options exist for NAFLD that target both sleep and NAFLD, including non-pharmacological factors, such as lifestyle modification (mainly diet and exercise). Pharmacological options include melatonin, Vitamin E, thiazolidinediones, and fecal microbiota transplantation.

\section{Core tip}

The pathogenesis of non-alcoholic fatty liver disease is closely tied to sleep and circadian rhythm abnormalities, through shared inflammatory pathways and altered metabolism. This review explores the pathogenesis of NAFLD in the context of sleep and circadian abnormalities. The associated inflammatory response is linked to changes in gut-microbiome interactions that contribute to the disease process. Understanding of this linkage has implications for various therapies for disease mitigation.

\section{More Information}

*Address for Correspondence: David A Johnson MD, MACG, FASGE, MACP, Professor of Medicine/Chief of Gastroenterology, Eastern VA Medical School, Norfolk, VA, USA, Tel: 757641 6685; Email: dajevms@aol.com

Submitted: August 04, 2021

Approved: August 16, 2021

Published: August 17, 2021

How to cite this article: D'Souza SM, Yoo BS, Houston KV, Patel AJ, Parekh PJ, et al. Nonalcoholic fatty liver disease: Update on treatment options and translational implications of sleep disruption. Ann Clin Gastroenterol Hepatol. 2021; 5: 032-038

DOI: 10.29328/journal.acgh.1001030

D'Souza SM: orcid.org/0000-0003-3772-2616 Yoo BS: orcid.org/0000-0002-8501-7922 Houston KV: orcid.org/0000-0002-8441-0132 Patel AJ: orcid.org/0000-0002-6981-3427 Parekh PJ: orcid.org/0000-0003-4750-775X Johnson DA: orcid.org/0000-0002-8737-0711

Copyright: (c) 2021 D'Souza SM, et al. This is an open access article distributed under the Creative Commons Attribution License, which permits unrestricted use, distribution, and reproduction in any medium, provided the original work is properly cited.

Keywords: Non-alcoholic fatty liver disease; Sleep; Circadian rhythms; Dysbiosis; Microbiome

Check for updates

OPEN ACCESS

\section{Introduction}

Fatty liver disease is a condition involving lipid accumulation within hepatocytes and has become the most common liver disease worldwide [1]. Non-alcoholic fatty liver disease (NAFLD) is a subset of fatty liver disease and is defined as hepatic steatosis without a cause of secondary fat accumulation such as excessive alcohol consumption, druginduced etiologies, or hereditary disorders [2]. This subset of fatty liver disease affects an estimated $30 \%$ of adults in the United States and a median of $20 \%$ adults worldwide [1].

\section{Pathogenesis of NAFLD}

Non-alcoholic fatty liver disease is further subdivided into non-alcoholic fatty liver (steatosis) and non-alcoholic steatohepatitis (NASH). The former is an isolated, nonalcoholic fatty liver that histologically involves greater than $5 \%$ of the liver parenchyma without inflammation or chronic hepatocyte injury [2]. This is in contrast to the latter, NASH, defined histologically as a necro-inflammatory process where greater than $5 \%$ of the liver parenchyma is involved and the hepatocytes become injured in a background of steatosis [2]. This process has been demonstrated to be progressive, with NASH leading to scarring, fibrosis and an increased risk of cirrhosis and hepatocellular carcinoma [3-5].

The pathogenesis of NAFLD involves the accumulation of fat, or lipids, within hepatocytes in the form of triglycerides. The primary reasons for this remain poorly understood, as some patients may have only isolated fat accumulation while 
others progress to NASH and have more progressive disease [6]. Current understanding involves alterations in metabolic pathways of hepatic lipid metabolism resulting from systemic insulin resistance, oxidative stress, and lipotoxicity [6]. A two-hit hypothesis helps to contextualize the current pathogenesis of NASH: the 'first-hit' consisting of excess hepatic triglyceride accumulation due to dysregulation of fatty acids and insulin resistance in the absence of other causes and the 'second-hit' consisting of oxidative stress and cytokine expression leading to a shift from steatosis to NASH $[7,8]$.

Sleep disruption has been a point of interest in regard to NAFLD, although the exact mechanisms have been poorly understood. Recent studies into NAFLD and various forms of sleep disruption in animal models and the transition to clinical practice will be explored in this article.

\section{Normal circadian function}

The human circadian rhythm is marked by the diurnal oscillation of function, affecting sleep, metabolism, and immune response [9-12]. The central regulator of circadian function is the suprachiasmatic nucleus (SCN) of the hypothalamus, which receives light-dark input from the retinohypothalamic tract to regulate circadian function through inhibition/activation of pineal gland production of melatonin, and through production and downstream effects of Circadian Locomotor Output Cycles Kaput (CLOCK) protein [13]. In addition, various tissues, including the liver and pancreas, display circadian automaticity in function, and are referred to as peripheral tissue oscillators [13]. Gastrointestinal circadian function is regulated by timing and frequency of food intake, and can affect the efficiency of nutrient absorption and processing, daily oscillation of gut microbial composition, and the related metabolic and immune consequences $[14,15]$. Clock protein dimerizes with Bmal1, and binds to an E-box, a DNA-response element site, to increase production of proteins including PER-1, PER-2, CRY1 , and CRY-2, which regulate various metabolic processes as well as provide periodic CLOCK gene inhibition $[16,17]$. Misalignment between central and peripheral rhythms, such as that seen in night-shift workers, are referred to as circadian dysrhythmia, and can be associated with loss of normal circadian function and metabolic derangements.

\section{OSA and Sleep Disruption in NAFLD}

Obstructive sleep apnea (OSA) has been well studied in its relationship to NAFLD. OSA is a sleep disorder characterized by repetitive episodes of non-breathing throughout the night, typically due to an upper airway obstruction or other anatomical abnormality and resulting in either hypopnea or apnea [18]. These episodes lead to wide ranging effects including chronic hypoxia during sleep or activation of the sympathetic nervous system and can have profound effects on the cardiovascular system, endocrine system, central nervous system [18]. Furthermore, this chronic hypoxia may lead to induction of oxidative stress and subsequent lipid peroxidation which could lead to hepatocyte necrosis and apoptosis, a has been postulated to be a key factor in the "second hit" in the pathogenesis of NAFLD [19].

Evidence has shown OSA to be a potential risk factor for the development and progression of NAFLD due to a hypoxia-induced mechanism [20,21]. Chronic hypoxia leads to oxidative stress, lipid peroxidation, and systemic inflammation leading to an imbalance between oxidant and antioxidant agents and a progression of NASH through liver fibrosis. One study evaluated patients with NAFLD through polysomnography and serum analysis [20]. The participants were divided into control, moderate OSA, and severe OSA. The results revealed a significant increase in alanine aminotransferase (ALT), aspartate aminotransferase (AST), total cholesterol, low density lipoprotein-cholesterol, and high-sensitivity C-reactive protein with increased severity of OSA. These findings were independent of factors such as age, gender, obesity, inflammation, blood pressure, serum glucose, and lipid profile suggesting nocturnal hypoxia as a risk factor in progression of NAFLD.

While the exact pathophysiologic mechanisms involved in the relationship of OSA and NAFLD are still being investigated, there have been several factors that seem to be at play in this multifactorial process. There is evidence that the chronic intermittent hypoxia may trigger liver injury, induce a systemic inflammatory state, and lead to fibrogenesis [22]. A key factor may be hypoxia-inducible factor 1-alpha (HIF1a), a transcription factor involved in the cellular response to hypoxia. One study comparing HIF-1a knockout to wild-type mice, after inducing NAFLD through a high trans-fat diet for 6 months, the wild-type mice had $80 \%$ more hepatic collagen [23]. Furthermore, this study induced the hepatocytes to a sustained hypoxic state vs normoxia and found that those under hypoxic conditions allowed for avid collagen crosslinking whereas knockout mice did not have significant cross-linking. Thus, hepatocyte HIF-1a appears to be a key mediator in the increase in hypoxia-induced liver fibrosis, and decrease may be protective from fibrosis [24]. HIF-1a activation seems to also have a close relationship to lectinlike oxidized low-density lipoprotein receptor-1 (LOX-1), a cell surface protein that is involved in endocytosis of lipoproteins into endothelial cells. It has been shown that LOX-1 levels are higher in patients with OSA compared to the normal population and this is important in regard to NAFLD pathogenesis because oxidative-LDL stimulates production of LOX-1 through a ROS/NF- $\kappa$ B signaling pathway and mediates ox-LDL induced endothelial injury and defenestration of human liver sinusoidal cells [25]. Thus it is reasonable to postulate the progression of NAFLD is mediated by a HIF$1 \mathrm{a}$ and its downstream effect on LOX-1 expression causing hepatocyte injury. 
Another study analyzed the association between chronic intermittent hypoxia $(\mathrm{CIH})$, morbid obesity, and the effect on liver and adipose tissue [21]. Across 101 morbidly obese subjects, histopathologic analysis of liver biopsies showed NAFLD lesions, NAFLD activity score, and fibrosis were significantly more severe in patients with higher oxygen desaturation index (a quantified severity of nocturnal $\mathrm{CIH}$ ). This suggests that there is a dose-response relationship between the amount of nocturnal oxygen desaturations and the severity of hepatic lesions found, all of which was independent of obesity and body composition [21]. A metaanalysis demonstrated OSA progressed in terms of liver enzymes and histological alterations and demonstrated OSA was independently associated with NAFLD developmen [19]. This evidence is in agreement that the chronic hypoxic state induced by the sleep disorder of OSA may be a key component as the second-hit in the pathogenesis of NAFLD. If left untreated, this hypoxia mechanism could lead to the progression from NAFLD to NASH.

\section{Sleep Insufficiency, Circadian Dysrhythmia, and NAFLD}

The direct effect of isolated sleep insufficiency and NAFLD has only recently been explored and has remained largely inconclusive [27,28]. In a large study published in 2013 of 69,463 middle-age workers and their spouses, one group assessed sleep duration and quality using the Pittsburgh Sleep Quality Index (PSQI) and then determined the presence of fatty liver [26]. Results showed that after controlling for confounding factors such as age, alcohol intake, smoking, and sleep apnea, with an odds ratio for NAFLD decrease in sleep duration of 1.28 in men and 1.71 in women. This implies that in the middle-aged general population, a shorter sleep duration and poor sleep quality are significantly associated with an increased risk of NAFLD. Various follow-up studies have demonstrated conflicting conclusions $[27,28]$. One study of Japanese subjects showed that short sleep duration of $<6$ hours was associated with reduced risk of NAFLD in men [27]. Another study of found no significant association with short sleep of $<7$ hours per day but found a significantly increased risk for NAFLD in those sleeping $>9$ hours per day [28]. The conflicting data on sleep duration and NAFLD, It is clear that work is needed to better understand the pathophysiologic mechanisms that may be involved regarding decreased sleep and NAFLD.

In addition to decrease in overall sleep time, alterations in sleep habits through circadian dysrhythmia can contribute to hepatic steatosis. One recent study evaluating the relationship between circadian misalignment and metabolic associated fatty liver disease (MAFLD) found prevalence of MAFLD to be $45 \%$ in the presence of circadian misalignment compared to $28 \%$ in the non-misaligned group [29]. The data also suggest that circadian misalignment is independently associated with MAFLD and fibrosis, while short sleep duration alone is not independently associated with this risk [29].

\section{Microbiome Changes, Sleep, and NAFLD}

The gut microbiome is an ecosystem within the human gut consisting of bacteria, fungi, and other microbes, which affect the host's physiology [30]. The microbiome is affected by the host's environmental factors such as diet, exercise, medications, circadian rhythm, and geographic location, and can also effect change in host physiology [30]. Environmental factors can lead to dysbiosis, a term used here to refer to a change in the composition of the microbiome that is associated with pathogenesis. The dysbiotic microbiome can alter activation of the immune system through direct interaction as well as indirectly through the production of metabolites that may trigger immune response locally or at other peripheral tissues through translocation. One such example would be through first-pass of these bacterial products to the liver through the portal system (gut-liver axis) [30-32]. Metabolic products such as phenols and ammonia along with proinflammatory bacterial components such as peptidoglycans and lipopolysaccharides (LPS) can trigger an inflammatory cascade that can lead to fibrosis $[32,33]$.

Several studies done in both humans and animals have demonstrated a link between gut dysbiosis and NAFLD [34-37]. One study examining non-obese patients with and without NAFLD, found a $20 \%$ increase in the phylum Bacteroidetes and a $24 \%$ decrease in the phylum Firmicutes among those with NAFLD [35]. Notably, flora capable of producing shortchain fatty acids (SCFA) and $7 \alpha$-dehydroxylation of bile acids were severely reduced [35]. Proteobacteria, another mainly gram negative phylum, have also been found to be elevated in patients with steatosis and NASH [36]. Another study evaluated stool samples of patients with biopsy-proven NAFLD using 16S ribosomal RNA gene sequencing, and found significant increase in Bacteroides spp. in patients with NASH and $\mathrm{F} \geq 2$ fibrosis [37]. Rumminococcus spp. were found to be increased within patients with severe fibrosis [37]. Changes in bacterial composition are linked to.

An important homeostatic mechanism would be the regulation on the amount and quality of the sleep the host is getting. As mentioned previously patients with NAFLD can have close associations with OSA and chronic intermittent hypoxia, which lead to dysbiosis, and a propensity to increase obligate anaerobes [38]. A one study on murine models, showed that a group receiving chronic intermittent hypoxia for 6 weeks, had a higher abundance of Firmicutes and a smaller abundance of Bacteroidetes and Proteobacteria phyla compared to the normoxic control [39]. Dysbiosis of the gut can lead to an increase in bacterial products such as reactive oxygen species or lipopolysaccharide which can ultimately circulate and cause increased inflammation within the liver.

\section{Role of therapeutics}

\section{Lifestyle modification}

Lifestyle modification including diet and exercise is 
the first line in managing patients with NAFLD. Primarily lifestyle modification is aimed at gradual weight loss by all main guidelines $[2,40,41]$. Various weight reduction targets have been proposed, including 3\% - 5\%, 5\% - 7\% and 7\% $10 \%$ weight loss for improvement of steatosis, inflammation, and NAFLD/NASH remission along with decrease in fibrosis, respectively [42]. One study of patients with histologically proven NASH evaluated 52 weeks of lifestyle modification for weight loss [43]. At the end of the 52 weeks, $25 \%$ of patients achieved resolution of steatohepatitis, $47 \%$ had reductions in NAFLD activity scores, and $19 \%$ had regression of fibrosis [43]. Patients with the highest amount of improvement had $\geq 10 \%$ overall weight loss [43]. While there is no specific diet currently recommended for NAFLD, the Mediterranean diet has shown improvement in other metabolic and cardiovascular diseases. Recently the ATTICA prospective observational study of healthy volunteers without any chronic diseases or diagnosis of NAFLD evaluated the role of a Mediterranean diet with routine follow-up in cardiovascular disease and metabolic disorders [44]. The study demonstrated that a Mediterranean diet was protective from diabetes mellitus and cardiovascular disease in patients with NAFLD [44]. Given the significant potential for benefit and low risk of harm, lifestyle modification through activity and dietary change remains a major recommendation for management of NAFLD.

\section{Melatonin}

Melatonin is a neurohormone that regulates the sleepwake cycle. Its production in the pineal gland is inhibited by light perception-mediated input from the SCN [45]. In addition to pineal production, several peripheral tissues produce and bind melatonin [46,47]. In addition to its role in mediating sleep function, melatonin has several immunomodulatory effects. Melatonin increases proliferation of several immune cell lines, including lymphocytes (particularly T-helper 1 cells), natural killer cells, and neutrophils [48]. Additionally, it acts to suppress the systemic inflammatory response through inhibition of the NLRP3 inflammasome, decreased nuclear translocation of pro-inflammatory transcription factor NF- $\mathrm{KB}$, anti-inflammatory transcription factor $\mathrm{Nrf2}$, and decreased macrophage toll-like receptor 4 signaling when activated by bacterial LPS [49,50]. In addition, melatonin has an overall anti-oxidant effect through scavenging by its metabolites as well as through up-regulation of superoxide dismutase and down-regulation of NADPH oxidase [51,52]. One study of patients with NAFLD found that melatonin supplementation over three months decreased weight, waist size, blood pressure, transaminases, and C-reactive protein, compared to baseline, however this did not reach statistical significance when compared to a control group [53]. In animal models, melatonin has been demonstrated to reduce expression of Micro-RNA-34a-5p, and inhibitor of sirtuin 1 production [54]. Increased sirtuin 1 availability has been linked to decreased hepatic inflammation and fibrosis [54]. In combination, supplementation of melatonin has potential for benefit through improved sleep as well as decreased propagation of the inflammatory cascade.

\section{Vitamin E}

Vitamin $\mathrm{E}$ is known to be a free radical scavenger, with use in patients with NASH without diabetes. In the pioglitazone, vitamin E or Placebo for the Treatment of Nondiabetic Patients with Nonalcoholic Steatohepatitis (PIVENS) trial, usage of $800 \mathrm{mg} /$ day was found to be superior to placebo in patients without diabetes and cirrhosis for improvement of NASH histology $43 \%$ vs. $19 \%$ respectively [55]. Another study of longer term use of vitamin E $(300 \mathrm{mg} /$ day) over 3 years demonstrated improved NASH fibrosis [56]. Currently, $300-400 \mathrm{mg}$ is being used rather than $800 \mathrm{mg}$ to decrease adverse effects of vitamin E toxicity [57]. Increased doses of vitamin $\mathrm{E}$ have been observed with increased all-cause mortality, hemorrhagic stroke and risk for prostatic cancer in individuals with a family history of prostate cancer [58-60].

\section{Thiazolidinediones}

Thiazolidinediones are peroxisome proliferator activated receptor (PPAR) $-\gamma$ ligands, which are used for type 2 diabetes mellitus (T2DM). Pioglitazone, a thiazolidinedione, has been shown in the past to significantly decrease steatosis and necroinflammation in patients with NASH and T2DM compared to placebos [61,62]. A meta-analysis showed improved steatosis, lobular inflammation, and ballooning on histology after usage of thiazolidinediones [63]. Thiazolidinediones have also been shown to improve fibrosis in NASH patients when compared to placebo, however it has not been seen in all studies to a significant extent [64-66]. A recent meta-analysis of 26 studies concluded that pioglitazone was associated with reductions in both AST and ALT and improved steatosis in both diabetic and non-diabetic NAFLD patients [67]. Pioglitazone is not without risk, it is well known to have a decently-sized side-effect profile ranging from weight gain, increased cardiovascular events, fluid retention, and increased risk for bone fractures in women $[40,67,68]$. While there is no finalized recommendation on dosing of pioglitazone, some groups recommend a safe starting dose of $15 \mathrm{mg}$ per day titrating up to $30 \mathrm{mg}$ per day for histologic improvement $[65,66,68]$. Further research is still being done on this drug group, as it shows promise for improvement on the histologic level.

\section{Fecal Microbiota transplantation}

Gut microbiome dysbiosis and NAFLD have a close association, and direct manipulation through fecal microbiota transplantation as a potential therapy has been proposed and demonstrated in murine models $[36,69]$. Compared to mice that were fed only HFD, mice that underwent FMT and fed 8 weeks of HFD demonstrated correction of gut microbiota 
with increased abundance of Christensenella spp. and Lactobacillus spp., significant reduction in intrahepatic lipid accumulation and intrahepatic pro-inflammatory cytokines, and improvement in steatohepatitis [69]. Developing from murine models, a double-blinded randomized controlled trial involving 21 patients who underwent allogenic or autologous FMT demonstrated that allogenic FMT resulted in a relative decrease in small intestinal permeability [70]. The intestinal membrane dysfunction has been associated with NAFLD, and reduction in the permeability may reflect a therapeutic potential in NAFLD [71]. Current clinical investigation is underway to evaluate the efficacy of FMT in NASH (NCT02469272).

\section{Conclusion}

Fatty liver disease and its complications are believed to be the result of altered metabolism as well as an associated cascade of systemic inflammation. Sleep-related disorders, including obstructive sleep apnea, insufficient sleep duration, and circadian dysrhythmia, can all contribute to NAFLD pathogenesis. This can occur through direct effects on central and peripheral circadian regulators or through changes in the diurnal oscillation of gut microbial composition. Nonpharmacological therapies, including lifestyle modification through diet and exercise, have demonstrated efficacy. Pharmacological therapies, including melatonin, vitamin E, thiazolidinediones, and fecal microbiota transplant, are all promising options.

\section{Author contribution}

Johnson DA, Parekh PJ, D' Souza SM and Yoo BS contributed construction of project; all authors wrote and edited the manuscript.

\section{References}

1. Vernon G, Baranova A, Younossi ZM. Systematic review: The epidemiology and natural history of non-alcoholic fatty liver disease and non-alcoholic steatohepatitis in adults. Aliment Pharmacol Ther. 2011; 34: 274-285.

PubMed: https://pubmed.ncbi.nlm.nih.gov/21623852/

2. Cani N, Younossi Z, Lavine JE, Charlton M, Cusi K, et al. The diagnosis and management of nonalcoholic fatty liver disease: Practice guidance from the American Association for the Study of Liver Diseases. Hepatology. 2018; 67: 328-357.

PubMed: https://pubmed.ncbi.nlm.nih.gov/28714183/

3. Loomba R, Wong R, Fraysse J, Shreay S, Li S, et al. Nonalcoholic fatty liver disease progression rates to cirrhosis and progression of cirrhosis to decompensation and mortality: a real world analysis of Medicare data. Aliment Pharmacol Ther. 2020; 51: 1149-1159. PubMed: https://pubmed.ncbi.nlm.nih.gov/32372515/

4. Chen K, Ma J, Jia X, Ai W, Ma Z, et al. Advancing the understanding of NAFLD to hepatocellular carcinoma development: From experimental models to humans. Biochim Biophys Acta - Rev Cancer. 2019; 1871: 117-125.

PubMed: https://pubmed.ncbi.nlm.nih.gov/30528647/

5. Diehl AM, Day C. Cause, Pathogenesis, and Treatment of Nonalcoholic Steatohepatitis. N Engl J Med. 2017; 377: 2063-2072. PubMed: https://pubmed.ncbi.nIm.nih.gov/29166236/
6. Angulo P. Nonalcoholic Fatty Liver Disease. N Engl J Med. 2002; 346: 1221-1231.

PubMed: https://pubmed.ncbi.nlm.nih.gov/11961152/

7. Farrell GC, Larter CZ. Nonalcoholic fatty liver disease: From steatosis to cirrhosis. Hepatology. 2006; 43: S99-S112. PubMed: https://pubmed.ncbi.nIm.nih.gov/16447287/

8. Browning JD, Horton JD. Molecular mediators of hepatic steatosis and liver injury. J Clin Invest. 2004; 114: 147-152.

PubMed: https://pubmed.ncbi.nlm.nih.gov/15254578/

9. Morris CJ, Yang JN, Garcia JI, Myers S, Bozzi I, et al. Endogenous circadian system and circadian misalignment impact glucose tolerance via separate mechanisms in humans. Proc Natl Acad Sci U S A. 2015; 112: E2225-E2234.

PubMed: https://pubmed.ncbi.nlm.nih.gov/25870289/

10. Eckel RH, Depner CM, Perreault L, Markwald RR, Smith MR, et al. Morning Circadian Misalignment during Short Sleep Duration Impacts Insulin Sensitivity. Curr Biol. 2015; 25: 3004-3010.

PubMed: https://pubmed.ncbi.nlm.nih.gov/26549253/

11. Carmo-Silva S, Cavadas C. Hypothalamic dysfunction in obesity and metabolic disorders. In: Advances in Neurobiology. Springer New York LLC. 2017; 19: 73-116.

PubMed: https://pubmed.ncbi.nIm.nih.gov/28933062/

12. Tarquini R, Mazzoccoli G. Clock Genes, Metabolism, and Cardiovascular Risk. Heart Fail Clin. 2017; 13: 645-655. PubMed: https://pubmed.ncbi.nIm.nih.gov/28865774/

13. Marcheva B, Ramsey KM, Peek CB, Affinati A, Maury E, et al. Circadian clocks and metabolism. Handb Exp Pharmacol. 2013; 217: 127-155. PubMed: https://pubmed.ncbi.nlm.nih.gov/23604478/

14. Panda S. Circadian physiology of metabolism. Science. 2016; 354: 1008-1015.

PubMed: https://pubmed.ncbi.nlm.nih.gov/27885007/

15. Poggiogalle $E$, Jamshed $H$, Peterson CM. Circadian regulation of glucose, lipid, and energy metabolism in humans. Metabolism. 2018; 84: 11-27.

PubMed: https://pubmed.ncbi.nlm.nih.gov/29195759/

16. Solt LA, Wang $Y$, Banerjee $S$, etal. Regulation of circadian behaviour and metabolism by REV-ERB- $\alpha$ and REV-ERB- $\beta$. Nature. 2012; 485: 62-68. PubMed: https://pubmed.ncbi.nlm.nih.gov/22460951/

17. Cho $H$, Zhao $X$, Hatori $M$, et al. Regulation of circadian behaviour and metabolism by REV-ERB- $\alpha$ and REV-ERB- $\beta$. Nature. 2012; 485: 123-127. PubMed: https://pubmed.ncbi.nlm.nih.gov/22460952/

18. Qaseem A, Dallas P, Owens DK, Starkey M, Holty J-EC, Shekelle P. Diagnosis of Obstructive Sleep Apnea in Adults: A Clinical Practice Guideline From the American College of Physicians. Ann Intern Med. 2014; 161: 210-220.

PubMed: https://pubmed.ncbi.nIm.nih.gov/25089864/

19. Jin S, Jiang S, Hu A. Association between obstructive sleep apnea and non-alcoholic fatty liver disease: a systematic review and metaanalysis. Sleep Breath. 2018; 22: 841-851.

PubMed: https://pubmed.ncbi.nlm.nih.gov/29335916/

20. Lin QC, Chen L Da, Chen GP, et al. Association between nocturnal hypoxia and liver injury in the setting of nonalcoholic fatty liver disease. Sleep Breath. 2015; 19: 273-280.

PubMed: https://pubmed.ncbi.nlm.nih.gov/24870112/

21. Aron-Wisnewsky J, Minville C, Tordjman J, et al. Chronic intermittent hypoxia is a major trigger for non-alcoholic fatty liver disease in morbid obese. J Hepatol. 2012; 56: 225-233.

PubMed: https://pubmed.ncbi.nlm.nih.gov/21703181/

22. Musso G, Olivetti C, Cassader M, Gambino R. Obstructive sleep apneahypopnea syndrome and nonalcoholic fatty liver disease: Emerging evidence and mechanisms. Semin Liver Dis. 2012; 32: 49-64. PubMed: https://pubmed.ncbi.nIm.nih.gov/22418888/ 
23. Mesarwi OA, Shin M-K, Bevans-Fonti S, Schlesinger C, Shaw J, Polotsky VY. Hepatocyte Hypoxia Inducible Factor-1 Mediates the Development of Liver Fibrosis in a Mouse Model of Nonalcoholic Fatty Liver Disease. Avila MA, ed. PLoS One. 2016; 11: e0168572. PubMed: https://pubmed.ncbi.nlm.nih.gov/28030556/

24. Moon JOK, Welch TP, Gonzalez FJ, Copple BL. Reduced liver fibrosis in hypoxia-inducible factor-1 $\alpha$-deficient mice. Am J Physiol Gastrointest Liver Physiol. 2009; 296: G582-592.

PubMed: https://pubmed.ncbi.nlm.nih.gov/19136383/

25. Akinnusi ME, Laporta R, El-Solh AA. Lectin-like oxidized low-density lipoprotein receptor-1 modulates endothelial apoptosis in obstructive sleep apnea. Chest. 2011; 140: 1503-1510.

PubMed: https://pubmed.ncbi.nlm.nih.gov/21565964/

26. Kim CW, Yun KE, Jung HS, Chang Y, Choi ES, et al. Sleep duration and quality in relation to non-alcoholic fatty liver disease in middleaged workers and their spouses. J Hepatol. 2013; 59: 351-357. PubMed: https://pubmed.ncbi.nlm.nih.gov/23578884/

27. Miyake T, Kumagi T, Furukawa S, Hirooka M, Kawasaki K, et al. Short sleep duration reduces the risk of nonalcoholic fatty liver disease onset in men: a community-based longitudinal cohort study. J Gastroenterol. 2015; 50: 583-589.

PubMed: https://pubmed.ncbi.nlm.nih.gov/25120172/

28. Liu C, Zhong R, Lou J, Pan A, Tang Y, et al. Nighttime sleep duration and risk of nonalcoholic fatty liver disease: the Dongfeng-Tongji prospective study. Ann Med. 2016; 48: 468-476.

PubMed: https://pubmed.ncbi.nlm.nih.gov/27327959/

29. Weng Z, Ou W, Huang J, Singh $M$, Wang $M$, et al. Circadian misalignment rather than sleep duration is associated with mafld: $A$ population-based propensity score-matched study. Nat Sci Sleep. 2021; 13: 103-111.

PubMed: https://pubmed.ncbi.nlm.nih.gov/33542668/

30. Kolodziejczyk AA, Zheng D, Shibolet O, Elinav E. The role of the microbiome in NAFLD and NASH . EMBO Mol Med. 2019; 11: e9302. PubMed: https://pubmed.ncbi.nlm.nih.gov/30591521/

31. Macpherson AJ, Heikenwalder M, Ganal-Vonarburg SC. The Liver at the Nexus of Host-Microbial Interactions. Cell Host Microbe. 2016; 20: 561-571.

PubMed: https://pubmed.ncbi.nlm.nih.gov/27832587/

32. Safari $Z$, Gérard $P$. The links between the gut microbiome and nonalcoholic fatty liver disease (NAFLD). Cell Mol Life Sci. 2019; 76: 1541-1558.

PubMed: https://pubmed.ncbi.nlm.nih.gov/30683985/

33. Milosevic I, Vujovic A, Barac A, Djelic M, Korac M, et al. Gut-liver axis, gut microbiota, and its modulation in the management of liver diseases: A review of the literature. Int J Mol Sci. 2019; 20: 395. PubMed: https://pubmed.ncbi.nlm.nih.gov/30658519/

34. Zhu L, Baker SS, Gill C, Liu W, Alkhouri R, et al. Characterization of gut microbiomes in nonalcoholic steatohepatitis (NASH) patients: A connection between endogenous alcohol and NASH. Hepatology. 2013; 57: 601-609.

PubMed: https://pubmed.ncbi.nlm.nih.gov/23055155/

35. Wang B, Jiang X, Cao M, et al. Altered fecal microbiota correlates with liver biochemistry in nonobese patients with non-alcoholic fatty liver disease. Sci Rep. 2016; 6: 1-11.

36. Aron-Wisnewsky J, Vigliotti C, Witjes J, Le P, Holleboom AG, et al. Gut microbiota and human NAFLD: disentangling microbial signatures from metabolic disorders. Nat Rev Gastroenterol Hepatol. 2020; 17:279-297. PubMed: https://pubmed.ncbi.nlm.nih.gov/32152478/

37. Boursier J, Mueller O, Barret M, Machado M, Fizanne L, et al. The severity of nonalcoholic fatty liver disease is associated with gut dysbiosis and shift in the metabolic function of the gut microbiota. Hepatology. 2016; 63: 764-775.

PubMed: https://pubmed.ncbi.nlm.nih.gov/26600078/
38. Leung C, Rivera L, Furness JB, Angus PW. The role of the gut microbiota in NAFLD. Nat Rev Gastroenterol Hepatol. 2016; 13: 412-425. PubMed: https://pubmed.ncbi.nlm.nih.gov/27273168/

39. Moreno-Indias I, Torres M, Montserrat JM, Sanchez-Alcoholado L, Cardona $\mathrm{F}$, et al. Intermittent hypoxia alters gut microbiota diversity in a mouse model of sleep apnoea. Eur Respir J. 2015; 45: 1055-1065. PubMed: https://pubmed.ncbi.nlm.nih.gov/25537565/

40. Polyzos SA, Kang ES, Boutari C, Rhee EJ, Mantzoros CS. Current and emerging pharmacological options for the treatment of nonalcoholic steatohepatitis. Metabolism. 2020; 111: 154203. PubMed: https://pubmed.ncbi.nlm.nih.gov/32151660/

41. Chalasani N, Younossi Z, Lavine JE, Diehl AM, Brunt EM, et al. The diagnosis and management of non-alcoholic fatty liver disease: Practice Guideline by the American Association for the Study of Liver Diseases, American College of Gastroenterology, and the American Gastroenterological Association. Hepatology. 2012; 55: 2005-2023. PubMed: https://pubmed.ncbi.nlm.nih.gov/22488764/

42. Hannah WN, Harrison SA. Effect of Weight Loss, Diet, Exercise, and Bariatric Surgery on Nonalcoholic Fatty Liver Disease. Clin Liver Dis. 2016; 20: 339-350.

PubMed: https://pubmed.ncbi.nlm.nih.gov/27063273/

43. Vilar-Gomez E, Martinez-Perez Y, Calzadilla-Bertot L, TorresGonzalez A, Gra-Oramas N, et al. Weight loss through lifestyle modification significantly reduces features of nonalcoholic steatohepatitis. Gastroenterology. 2015; 149: 367-378.e5. PubMed: https://pubmed.ncbi.nlm.nih.gov/25865049/

44. Kouvari M, Boutari C, Chrysohoou C, Fragkopoulou E, Antonopoulou $\mathrm{S}$, et al. Mediterranean diet is inversely associated with steatosis and fibrosis and decreases ten-year diabetes and cardiovascular risk in NAFLD subjects: Results from the ATTICA prospective cohort study. Clin Nutr. 2021; 40: 3314-3324.

PubMed: https://pubmed.ncbi.nlm.nih.gov/33234342/

45. Borjigin J, Samantha Zhang L, Calinescu AA. Circadian regulation of pineal gland rhythmicity. Mol Cell Endocrinol. 2012; 349: 13-19. PubMed: https://pubmed.ncbi.nlm.nih.gov/21782887/

46. Gao J, Xu K, Liu H, Liu G, Bai M, et al. Impact of the Gut Microbiota on Intestinal Immunity Mediated by Tryptophan Metabolism. Front Cell Infect Microbiol. 2018; 8: 13

PubMed: https://pubmed.ncbi.nlm.nih.gov/29468141/

47. Thor PJ, Krolczyk G, Gil K, Zurowski D, Nowak L. Melatonin and serotonin effects on gastrointestinal motility. J Physiol Pharmacol An Off J Polish Physiol Soc. 2007; 58 Suppl 6: 97-103. PubMed: https://pubmed.ncbi.nlm.nih.gov/18212403/

48. Miller SC, Pandi PSR, Esquifino Al, Cardinali DP, Maestroni GJM. The role of melatonin in immuno-enhancement: Potential application in cancer. Int J Exp Pathol. 2006; 87: 81-87. PubMed: https://pubmed.ncbi.nlm.nih.gov/16623752/

49. Bonomini F, Dos Santos M, Veronese FV, Rezzani R. NLRP3 inflammasome modulation by melatonin supplementation in chronic pristane-induced lupus nephritis. Int J Mol Sci. 2019; 20: 3466. PubMed: https://pubmed.ncbi.nlm.nih.gov/31311094/

50. Tarocco A, Caroccia N, Morciano G, Wieckowski MR, Ancora G, et al. Melatonin as a master regulator of cell death and inflammation: molecular mechanisms and clinical implications for newborn care. Cell Death Dis. 2019; 10: 317

PubMed: https://pubmed.ncbi.nlm.nih.gov/30962427/

51. Galano A, Tan DX, Reiter RJ. Melatonin: A versatile protector against oxidative DNA damage. Molecules. 2018; 23: 530. PubMed: https://pubmed.ncbi.nlm.nih.gov/29495460/

52. Zhou J, Zhang S, Zhao X, Wei T. Melatonin impairs NADPH oxidase assembly and decreases superoxide anion production in microglia exposed to amyloid-B1-42. J Pineal Res. 2008; 45: 157-165. PubMed: https://pubmed.ncbi.nlm.nih.gov/18298462/ 
53. Pakravan H, Ahmadian M, Fani A, Aghaee D, Brumanad S, et al. The Effects of Melatonin in Patients with Nonalcoholic Fatty Liver Disease: A Randomized Controlled Trial. Adv Biomed Res. 2017; 6: 40. PubMed: https://pubmed.ncbi.nlm.nih.gov/28503495/

54. Stacchiotti A, Grossi I, García-Gómez R, Patel GA, Salvi A, et al. Melatonin Effects on Non-Alcoholic Fatty Liver Disease Are Related to MicroRNA-34a-5p/Sirt1 Axis and Autophagy. Cells. 2019; 8: 1053. PubMed: https://pubmed.ncbi.nlm.nih.gov/31500354/

55. Sanyal AJ, Chalasani N, Kowdley KV, McCullough A, Diehl AM, et al. Pioglitazone, Vitamin E, or Placebo for Nonalcoholic Steatohepatitis. N Engl J Med. 2010; 362: 1675-1685.

PubMed: https://pubmed.ncbi.nlm.nih.gov/20427778/

56. Sumida $Y$, Naito $Y$, Tanaka S, Sakai $K$, Inada $Y$, et al. Long-term $(\geq 2 \mathrm{yr})$ efficacy of vitamin $E$ for non-alcoholic steatohepatitis. Hepatogastroenterology. 2013; 60: 1445-1450.

PubMed: https://pubmed.ncbi.nlm.nih.gov/23933938/

57. Rinella ME, Sanyal AJ. Management of NAFLD: A stage-based approach. Nat Rev Gastroenterol Hepatol. 2016; 13: 196-205. PubMed: https://pubmed.ncbi.nlm.nih.gov/26907882/

58. Miller ER, Pastor-Barriuso R, Dalal D, Riemersma RA, Appel LJ, Guallar E. Meta-analysis: High-dosage vitamin E supplementation may increase all-cause mortality. Ann Intern Med. 2005; 142: 37-46. PubMed: https://pubmed.ncbi.nlm.nih.gov/15537682/

59. Schürks M, Glynn RJ, Rist PM, Tzourio C, Kurth T. Effects of vitamin $\mathrm{E}$ on stroke subtypes: Meta-analysis of randomised controlled trials. BMJ. 2010; 341: c5702.

PubMed: https://pubmed.ncbi.nlm.nih.gov/21051774/

60. Lippman SM, Klein EA, Goodman PJ, Lucia MS, Thompson IM, et al. Effect of selenium and vitamin $E$ on risk of prostate cancer and other cancers: The selenium and vitamin $\mathrm{E}$ cancer prevention trial (SELECT). JAMA - J Am Med Assoc. 2009; 301: 39-51.

PubMed: https://pubmed.ncbi.nlm.nih.gov/19066370/

61. Belfort R, Harrison SA, Brown K, Darland C, Finch J, et al. A PlaceboControlled Trial of Pioglitazone in Subjects with Nonalcoholic Steatohepatitis. N Engl J Med. 2006; 355: 2297-2307. PubMed: https://pubmed.ncbi.nlm.nih.gov/17135584/

62. Aithal GP, Thomas JA, Kaye PV, Lawson A, Ryder SD, et al. Randomized, Placebo-Controlled Trial of Pioglitazone in Nondiabetic Subjects With Nonalcoholic Steatohepatitis. Gastroenterology. 2008; 135: 1176-1184.

PubMed: https://pubmed.ncbi.nlm.nih.gov/18718471/
63. Singh S, Khera R, Allen AM, Murad MH, Loomba R. Comparative effectiveness of pharmacological interventions for nonalcoholic steatohepatitis: A systematic review and network meta-analysis. Hepatology. 2015; 62: 1417-1432.

PubMed: https://pubmed.ncbi.nlm.nih.gov/26189925/

64. Cusi K, Orsak B, Bril F, Lomonaco R, Hecht J, et al. Long-term pioglitazone treatment for patients with nonalcoholic steatohepatitis and prediabetes or type 2 diabetes mellitus a randomized trial. Ann Intern Med. 2016; 165: 305-315.

PubMed: https://pubmed.ncbi.nlm.nih.gov/27322798/

65. Bril F, Biernacki DM, Kalavalapalli S, Lomonaco R, Subbarayan SK, et al. Role of Vitamin $E$ for nonalcoholic steatohepatitis in patients with type 2 diabetes: A randomized controlled trial. Diabetes Care. 2019; 42: 1481-1488.

PubMed: https://pubmed.ncbi.nlm.nih.gov/31332029/

66. Budd J, Cusi K. Role of Agents for the Treatment of Diabetes in the Management of Nonalcoholic Fatty Liver Disease. Curr Diab Rep. 2020; 20: 59 .

PubMed: https://pubmed.ncbi.nlm.nih.gov/33015726/

67. Kumar J, Memon RS, Shahid I, Rizwan T, Zaman M, et al. Antidiabetic drugs and non-alcoholic fatty liver disease: A systematic review, meta-analysis and evidence map. Dig Liver Dis. 2021; 53: 44-51. PubMed: https://pubmed.ncbi.nlm.nih.gov/32912770/

68. Sumida $Y$, Yoneda $M$. Current and future pharmacological therapies for NAFLD/NASH. J Gastroenterol. 2018; 53: 362-376. PubMed: https://pubmed.ncbi.nlm.nih.gov/29247356/

69. Zhou D, Pan Q, Shen F, Cao HX, Ding WJ, et al. Total fecal microbiota transplantation alleviates high-fat diet-induced steatohepatitis in mice via beneficial regulation of gut microbiota. Sci Rep. 2017; 7: 1529. PubMed: https://pubmed.ncbi.nlm.nih.gov/28484247/

70. Craven L, Rahman A, Nair Parvathy S, Beaton M, Silverman J, et al. Allogenic Fecal Microbiota Transplantation in Patients with Nonalcoholic Fatty Liver Disease Improves Abnormal Small Intestinal Permeability: A Randomized Control Trial. Am J Gastroenterol. 2020; 115: 1055-1065.

PubMed: https://pubmed.ncbi.nlm.nih.gov/32618656/

71. Citi S. Intestinal barriers protect against disease: Leaky cell-cell junctions contribute to inflammatory and autoimmune diseases. Science. 2018; 359: 1097-1098.

PubMed: https://pubmed.ncbi.nlm.nih.gov/29590026/ 